\title{
Mikrokontakt-Strukturierung von Platindünnschichten als Elektrodenmaterialien
}

\author{
Florian M. Wisser ${ }^{1}$, Julia Grothe ${ }^{1}$, Stefan Kaskel ${ }^{1}$ \\ ${ }^{1}$ Technische Universität Dresden \\ Fakultät Mathematik und Naturwissenschaften \\ Fachrichtung Chemie und Lebensmittelchemie \\ Professur Anorganische Chemie I \\ Bergstraße 66 \\ 01069 Dresden
}

Julia.Grothe@chemie.tu-dresden.de

\begin{abstract}
:
Strukturierte Platinelektroden wurden durch Mikrokontaktdrucken eines polymeren Präkursors hergestellt. Der Präkursor besteht aus einem anpolymerisiertem Zitronensäure-Ethylenglycolester und Platinnitrat in verdünnter Salpetersäure. Die so hergestellten Interdigitalelektroden mit einer lateralen Auflösung von $20 \mu \mathrm{m}$ wurden röntgenographisch und elektrochemisch charakterisiert. Die elektrochemische Gehaltsbestimmung von Wasserstoffperoxid ist mit diesen Platinelektroden ohne zusätzliche Referenzelektrode bis zu einem Gehalt von etwa 0,5 ppm (10 $\mu \mathrm{M})$ möglich.
\end{abstract}

Schlüsselbegriffe: Platin, Dünnschicht, Mikrokontaktdrucken, Interdigitalelektrode, elektrochemische Wasserstoffperoxid-Bestimmung

\section{Einleitung}

Edelmetallelektroden, insbesondere Platinelektroden werden auf Grund ihrer chemischen Stabilität in einer Vielzahl elektronischer Bauteile wie Brennstoffzellen, (Hochtemperatur)Sensoren oder Solarzellen verwendet.[1-6] Die eingesetzten Elektroden werden dabei meist über Siebdrucktechnik, Fotolithographie, Magnetronsputtern oder durch Strukturierung von kompakten Schichten mittels fokussierter Ionen- oder Elektronenstrahlen hergestellt.[7-9] Bei erstgenannten Methoden werden relativ hohe Strukturen mit in der Regel recht großen Breiten deutlich über $50 \mu \mathrm{m}$ erzeugt, bei letzteren wird ein Teil des Edelmetalls während des Strukturierens verdampft. Banks und Mitarbeiter benutzen mittels Siebdruck hergestellte Platinelektroden zur elektrochemischen Detektion von Wasserstoffperoxid im Konzentrationsbereich von 100 bis $1000 \mu \mathrm{M}$.[6]

Um den Materialeinsatz sowie die laterale Auflösung der Elektroden unter Beibehaltung der elektrochemischen Eigenschaften zu verrin- gern, wurde angestrebt, diese über Mikrokontaktdrucken $(\mu-\mathrm{CP})$ geeigneter Vorstufen herzustellen.

Durch die Verwendung eines auf einem Zitronensäure-Ethylenglycolester basierenden Platinpräkursors konnten mittels des $\mu$-CPs Interdigitalelektroden (IDE) hergestellt werden. Diese konnten als Elektroden in einem elektrochemischen Sensor zur Detektion von Wasserstoffperoxid eingesetzt werden.

\section{Experimentelles}

Ethylenglycol $(99,5 \%)$ und Salpetersäure $(69 \%)$ wurden von Sigma Aldrich, Platin(II)nitrat $(57,81 \% \mathrm{Pt})$ von ChemPur und Zitronensäure (99\%, wasserfrei) von Acros Organics bezogen.

\section{Platinpräkursor}

Der Platinpräkursor wurde gemäß der in der Literatur beschriebenen Vorschrift hergestellt.[10] Dafür wurden $5,830 \mathrm{~g}(30,3 \mathrm{mmol})$ Zitronensäure bei $90^{\circ} \mathrm{C}$ unter Rühren in 
$3,754 \mathrm{~g}(60,5 \mathrm{mmol})$ Ethylenglycol gelöst. Nach dem vollständigen Lösen wurden $6 \mathrm{~mL}$ einer halbkonzentrierten Salpetersäure hinzugegeben. Nach einer Stunde wurde der oligomere Präkursor auf Raumtemperatur abgekühlt. In einem Zehntel der Lösung wurden $373,3 \mathrm{mg}$ $(1,1 \mathrm{mmol})$ Platin(II)nitrat Monohydrat gelöst.

\section{Herstellung dünner Schichten}

Zur Herstellung der Dünnschichten (als Dünnschicht werden hier Filme mit einer Dicke zwischen 0,1 und $100 \mathrm{~nm}$ bezeichnet) wurde ein Tropfen des Platinpräkursors auf ein gereinigtes Glassubstrat aufgebracht und per Schleuderbeschichtung ein kompakter Präkursorfilm erzeugt. Die kompakten Platindünnschichten wurden durch thermische Zersetzung der organischen Bestandteile bei $600^{\circ} \mathrm{C}$ (Heizrate: $5 \mathrm{~K} / \mathrm{min}$ ) hergestellt.[10]

\section{Mikrokontaktdrucken}

Das Mikrokontaktdrucken wurde mit Hilfe eines Micro-Contact-Printing System $\mu$-CP 3.0 der Firma GeSiM durchgeführt. Dazu wurde ein strukturierter PDMS-Stempel kontrolliert in den Präkursorfilme gedrückt und dieser thermisch durch Heizen auf $90^{\circ} \mathrm{C}$ ausgehärtet. Nach dem Härten wurde der Stempel entfernt und durch tempern bei $600^{\circ} \mathrm{C}$ (Heizrate: $5 \mathrm{~K} / \mathrm{min}$ ) die organsichen Bestandteile zersetzt.

\section{Analytik}

Elektrochemische Messungen wurden an einem IVIUMSTAT electrochemical interface (Ivium technologies) durchgeführt (Scangeschwindigkeit: $50 \mathrm{mV} / \mathrm{s}$ ). Als Referenzelektrode wurde eine gesättigte Kalomelelektrode verwendet. Die Messungen für die Wasserstoffperoxid-Bestimmungen wurden bei einem konstanten Potential von 0,2 V gegen Platin durchgeführt, der Messwert wurde nach $120 \mathrm{~s}$ bestimmt. Der Flächenwiderstand wurde mit einer Vierpunktmessanordnung der Firma Cascade Microtech mit einem Keithley 2400 source meter bestimmt. Röntgendiffraktogramme wurden an einem PANalytical X'Pert PRO Diffraktometer gemessen (Bragg-Brentano Geometrie, $\mathrm{Cu}_{\mathrm{ka} 1}$ Strahlung). REM- und EDXAufnahmen wurden an einem Zeiss DSM-982 Gemini angefertigt.

\section{Ergebnisse}

\section{Platindünnschichten}

Durch Tempern bei $600{ }^{\circ} \mathrm{C}$ konnten phasenreine Platindünnschichten erhalten werden. Diese wiesen bei einer mittleren Dicke von $57 \mathrm{~nm}$ einen mittleren spezifischen Widerstand von etwa $1,7 \cdot 10^{-7} \Omega \mathrm{m}$. Der geringste spezifische
Widerstand lag mit $1,1 \cdot 10^{-7} \Omega \mathrm{m}$ (Schichtwiderstand: $0,59 \Omega, 42 \mathrm{~nm}$ ) nur geringfügig über dem des Bulkmaterials mit $1,05 \cdot 10^{-7} \Omega \mathrm{m} \cdot[10,11]$

\section{Strukturierte Elektroden}

Die hier untersuchten IDE bestehen aus Fingern mit einer Breite von etwa $20 \mu \mathrm{m}$ (Abb. 1), die ebenfalls aus röntgenographisch phasenreinem Platin bestehen (Abb. 2). Durch das Drucken des Stempels in eine kompakte Schicht verbleibt stets eine sehr dünne Restschicht Platin zwischen zwei Fingern. Diese ist jedoch so dünn, dass sie mittels EDX nicht erfasst werden kann (Abb. 1).

Wie bereits in unserer vorherigen Veröffentlichung gezeigt, weisen Linien mit einer Breite von $15 \mu \mathrm{m}$ über die Druckfläche von $1 \times 1 \mathrm{~cm}^{2}$ einen Widerstand von etwa $100 \Omega$ auf.[10] Dies entspricht etwa einem spezifischen Widerstand von $2,0 \cdot 10^{-5} \Omega \mathrm{m}$. Der Widerstand der Finger der IDE sollte im gleichen Bereich liegen.

Um zu zeigen, dass die einzelnen Finger untereinander nicht elektrisch kontaktiert sind, wurden Cyclovoltammogramme in $1 \mathrm{M}$ Perchlorsäure aufgenommen. Diese zeigen stets ein für Platin typisches Cyclovoltammogramm mit der Wasserstoffadsorption bzw. desorption im Bereich von 0 bis $-0,25 \mathrm{~V}$ sowie die Oxidation und anschließende Reduktion von Platin zwischen 0,4 und $1,25 \mathrm{~V}$ bzw. 0,8 und $0,2 \vee$ (Abb. 3).[12]

Bei Verwendung einer Zwei-ElektrodenAnordnung, bei der ein Finger der Elektrode als Arbeits-, der andere als Gegen- und Referenzelektrode verwendet wurde, beginnt die anodische Zersetzung des Wassers bereits bei etwa einem Potential von 0,8 V. Zwischen -0,25 und $0,8 \mathrm{~V}$ im sauren Milieu finden jedoch keine Redoxreaktionen statt (Abb. 3).

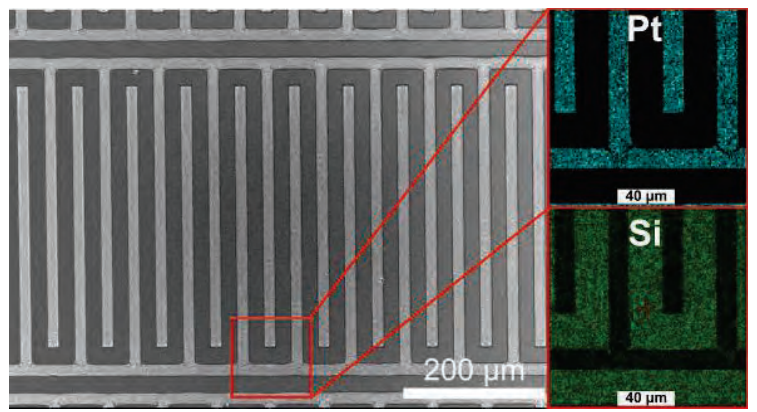

Abb. 1: links: REM-Aufnahme einer Platin-IDE, rechts dazugehörige EDX-Analysen. 


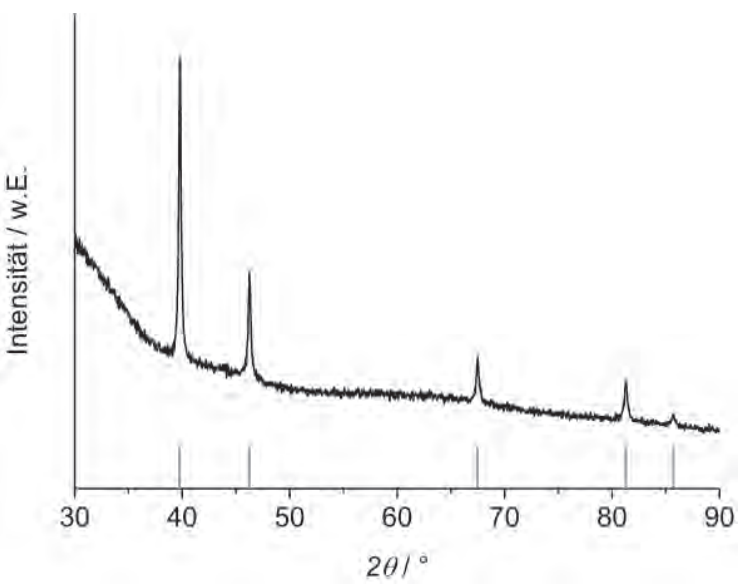

Abb. 2: Röntgendiffraktogramm der Platin-IDE (schwarz), Bragg-Markierungen von Platin (ICSD: 01-089-7382, grau).

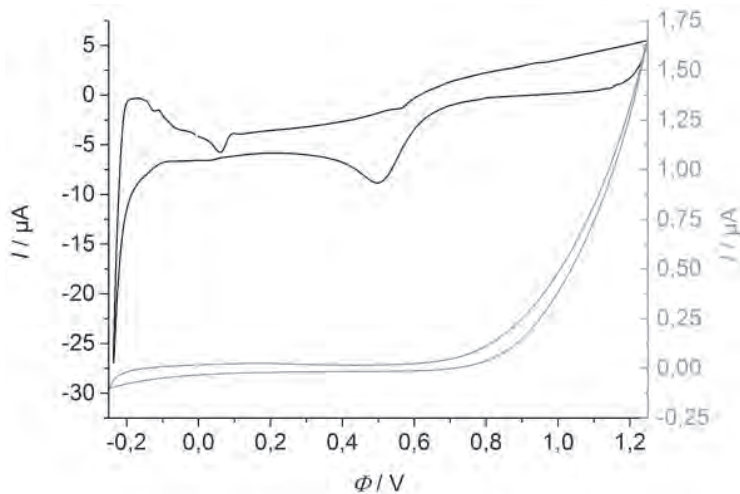

Abb. 3: Cyclovoltammogramm einer IDE in $1 \mathrm{M}$ $\mathrm{HClO}_{4}$ : Drei-Elektroden-Anordnung (schwarz), ZweiElektroden-Anordnung (grau).

\section{Wasserstoffperoxid-Bestimmung}

In einem Phosphatpuffer bei $\mathrm{pH} 7$ findet die kathodische Reduktion des Wassers bei Potentialen unterhalb von $-0,5 \mathrm{~V}$ statt, ebenfalls in der Zwei-Elektroden-Anordnung gemessen (Abb. 4). Somit ist der Bereich, indem keine Redoxreaktion stattfindet, im Vergleich zum sauren Milieu ausgeweitet. Eine $1 \mathrm{mM}$ Wasserstoffperoxid-Lösung zeigt eine Oxidation bereits bei $0,2 \vee(A b b .4)$. Für die amperometrische Wasserstoffperoxid-Bestimmung wurde das Potential der Elektroden daher auf $0,2 \mathrm{~V}$ eingestellt. Im Bereich von 10 bis $750 \mu \mathrm{M}$ Wasserstoffperoxid zeichnen sich die Elektroden durch eine lineare Korrelation zwischen Strom und Konzentration aus:

$I=14,603 \mathrm{nA}+0,627 \cdot c \cdot \mathrm{nA} / \mu \mathrm{M}\left(\mathrm{R}^{2}=0,996\right)$.

Bei $1000 \mu \mathrm{M}$ Wasserstoffperoxid weicht der Strom schon deutlich vom linearen Verhalten ab (Abb. 5). Im Vergleich zu mittels Siebdruck hergestellten Elektroden kann bei den Interdigitalelektroden zum einen auf eine zusätzliche Referenzelektrode verzichtet werden, zum an- deren konnte das untere Ende des Messbereiches um eine Zehnerpotenz gesenkt werden.[6]

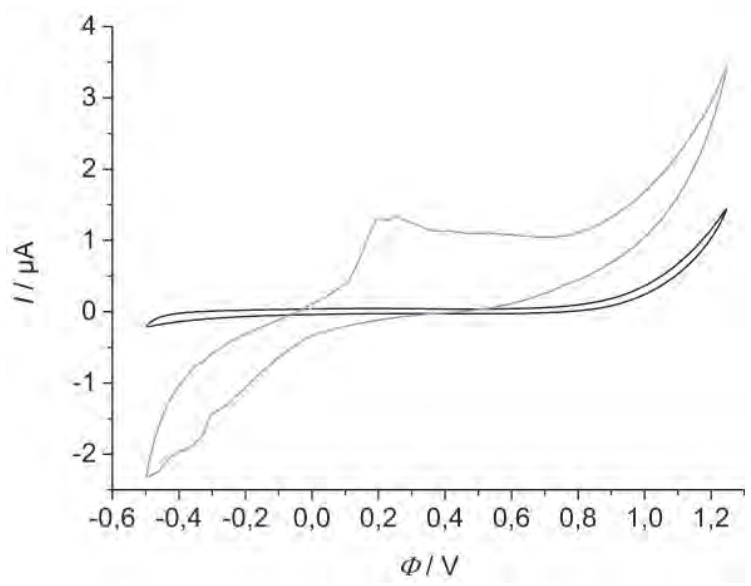

Abb. 4: Cyclovoltammogramm der IDE im Phosphatpuffer bei pH7 vor (schwarz) und nach (grau) der Zugabe von $1 \mathrm{mM} \mathrm{H}_{2} \mathrm{O}_{2}$.

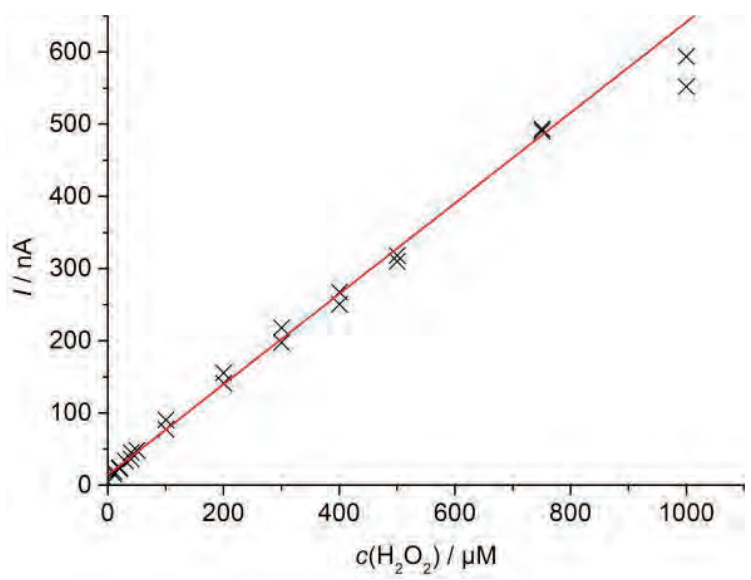

Abb. 5: Kalibrierkurve der WasserstoffperoxidBestimmung.

\section{Zusammenfassung}

Erstmalig konnten Platininterdigitalelektroden durch ein direktes Druckverfahren hergestellt werden. Diese sind röntgenographisch phasenrein und zeigen im wässrigen Milieu ein Redoxverhalten, das vergleichbar mit dem des Bulkmaterials ist.

Es konnte gezeigt werden, dass die IDE sowohl in einer Drei- als auch in einer Zwei-ElektrodenAnordnung verwendet werden können. Letzteres hat den Vorteil, dass keine zusätzliche Referenzelektrode in das System eingebracht werden muss. Dadurch ist ein kompakterer Aufbau der Messzelle möglich. Darüber hinaus konnten die IDE in der elektrochemischen Wasserstoffperoxid-Bestimmung ebenfalls ohne zusätzliche Referenzelektrode eingesetzt werden. Im Bereich von 10 bis $750 \mu \mathrm{M}$ (etwa 0,5 bis 40 ppm) weisen die Elektroden eine ausgezeichnete lineare Korrelation zwischen Konzentration und gemessenem Strom auf. 


\section{Literatur}

[1] S. Chatterjee and A. Chen, Electrochem. Commun. 20, 2012, 29-32; doi: 10.1016/j.elecom.2012.03.044

[2] S. Ito, T. N. Murakami, P. Comte, P. Liska, C. Grätzel, M. K. Nazeeruddin and M. Grätzel, Thin Solid Films 516, 2008, 46134619; doi: 10.1016/j.tsf.2007.05.090

[3] M. W. Louie and S. M. Haile, Energy Environ. Sci. 4, 2011, 4230-4238; doi: 10.1039/c1ee01889b

[4] A. Jiang and T. Tang, Ceram. Int. 38, 2012, 69-72; doi: 10.1016/j.ceramint.2011.04.052

[5] P. Kirchner, J. Oberländer, P. Friedrich, J. Berger, G. Rysstad, M. Keusgen and M. J. Schöning, Sens. Actuators B 170, 2012 60-66; doi: 10.1016/j.snb.2011.01.032

[6] J. P. Metters, F. Tan, R. O. Kadara and C. E. Banks, Anal. Methods 4, 2012, 12721277; doi: 10.1039/c2ay05934g

[7] L. Rotkina, S. Oh, J. N. Eckstein and S. V. Rotkin, Phys. Rev. B: Condens. Matter Mater. Phys. 72, 2005, 233407; doi: 10.1103/PhysRevB.72.233407

[8] J.-F. Lin, J. P. Bird, L. Rotkina and P. A. Bennett, Appl. Phys. Lett. 82, 2003, 802804; doi: 10.1063/1.1541940

[9] P. Greco, M. Cavallini, P. Stoliar, S. D. Quiroga, S. Dutta, S. Zacchini, M. C. lapalucci, V. Morandi, S. Milita, P. G. Merli and F. Biscarini, J. Am. Chem. Soc. 130, 2008, 1177-1182; doi: 10.1021/ja074104m

[10] F. M. Wisser, B. Schumm, A. Meier, T. Engel, J. Grothe, G. Kickelbick, S. Kaskel, J. Mater. Chem. C 13, 2013, 2477-2484; doi: $10.1039 / \mathrm{c} 3 \mathrm{tc00826f}$

[11] J. Goswami, C.-G. Wang, W. Cao and S. K. Dey, Chem. Vap. Deposition 9, 2003, 213220, doi: $10.1002 /$ cvde. 200306240

[12] R. G. Freitas, R. T. S. Oliveira, M. C. Santos, L. O. S. Bulhões and E. C. Pereira, Mater. Lett. 60, 2006, 1906-1910; doi : 10.1016/j.matlet.2005.12.050 